\title{
Characteristics of EUV Coronal Jets Observed with STEREO/SECCHI
}

\author{
G. Nisticò · V. Bothmer · S. Patsourakos · G. Zimbardo
}

Received: 16 February 2009 / Accepted: 25 July 2009 / Published online: 3 September 2009

(c) The Author(s) 2009. This article is published with open access at Springerlink.com

\begin{abstract}
In this paper we present the first comprehensive statistical study of EUV coronal jets observed with the SECCHI (Sun Earth Connection Coronal and Heliospheric Investigation) imaging suites of the two STEREO spacecraft. A catalogue of 79 polar jets is presented, identified from simultaneous EUV and white-light coronagraph observations, taken during the time period March 2007 to April 2008, when solar activity was at a minimum. The twin spacecraft angular separation increased during this time interval from 2 to 48 degrees. The appearances of the coronal jets were always correlated with underlying small-scale chromospheric bright points. A basic characterization of the morphology and identification of the presence of helical structure were established with respect to recently proposed models for their origin and temporal evolution. Though each jet appeared morphologically similar in the coronagraph field of view, in the sense of a narrow collimated outward flow of matter, at the source region in the low corona the jet showed different characteristics, which may correspond to different magnetic structures. A classification of the events with respect to previous jet studies shows that amongst the 79 events there were 37 Eiffel tower-type jet
\end{abstract}

STEREO Science Results at Solar Minimum

Guest Editors: Eric R. Christian, Michael L. Kaiser, Therese A. Kucera, O.C. St Cyr.

G. Nisticò · G. Zimbardo ( $₫)$

Dipartimento di Fisica, Università della Calabria, Ponte P. Bucci Cubo 31C, 87036, Rende, Italy

e-mail: zimbardo@fis.unical.it

G. Nisticò

e-mail: nisticogiuseppe @libero.it

V. Bothmer

Göttingen University, Institut für Astrophysik, Göttingen, Germany

e-mail: bothmer@astro.physik.uni-goettingen.de

S. Patsourakos

Naval Research Laboratory, Washington, DC, USA

e-mail: spatsourakos@ssd5.nrl.navy.mil

S. Patsourakos

Center for Earth Observing and Space Research, College of Science, George Mason University, Fairfax, VA 22030, USA 
events, commonly interpreted as a small-scale ( $\sim 35$ arc sec) magnetic bipole reconnecting with the ambient unipolar open coronal magnetic fields at its loop tops, and 12 lambda-type jet events commonly interpreted as reconnection with the ambient field happening at the bipole footpoints. Five events were termed micro-CME-type jet events because they resembled the classical coronal mass ejections (CMEs) but on much smaller scales. The remaining 25 cases could not be uniquely classified. Thirty-one of the total number of events exhibited a helical magnetic field structure, indicative for a torsional motion of the jet around its axis of propagation. A few jets are also found in equatorial coronal holes. In this study we present sample events for each of the jet types using both, STEREO A and STEREO B, perspectives. The typical lifetimes in the SECCHI/EUVI (Extreme UltraViolet Imager) field of view between 1.0 to $1.7 R_{\odot}$ and in SECCHI/COR1 field of view between 1.4 to $4 R_{\odot}$ are obtained, and the derived speeds are roughly estimated. In summary, the observations support the assumption of continuous small-scale reconnection as an intrinsic feature of the solar corona, with its role for the heating of the corona, particle acceleration, structuring and acceleration of the solar wind remaining to be explored in more detail in further studies.

Keywords Coronal holes $\cdot$ Helicity, observations $\cdot$ Magnetic reconnection, observational signatures $\cdot$ Jets

\section{Introduction}

The twin spacecraft making up the STEREO (Solar TErrestrial RElations Observatory) mission have already provided a rich dataset since launch in October 2006, with images both in the visible and in the Extreme UltraViolet (EUV) (Kaiser et al., 2008). These images are collected by the EUVI (Extreme UltraViolet Imager) A and B imager and COR1 (CORonagraph1) and COR2 A and B coronagraphs on the STEREO A and B satellites as part of the SECCHI (Sun-Earth Connection Coronal and Heliospheric Investigation) instrument packages (Howard et al., 2008). Each SECCHI suite consists of the following five telescopes: the EUVI imager and COR1 and COR2 coronagraphs comprise the SCIP package pointing to the Sun, complemented by the off-pointing HI1 (Heliospheric Imagerl) and HI2 imagers observing the heliosphere. All imagers together cover the observational range from the Sun to beyond $1 \mathrm{AU}$.

Besides the classical coronal mass ejections (CMEs) that are identified in the FOVs of the COR imagers (see e.g. Howard and Tappin, 2008), small-scale collimated ejections of solar plasma are another common feature (see e.g. Patsourakos et al., 2008). These coronal jets are best observed inside polar coronal holes at EUV wavelengths when the plasma beams are seen in emission against the dark background and are not obscured by bright ambient coronal structures. Very early observations of coronal jets were provided by $S k y$ $l a b$ and, in more detail, later by Yohkoh in soft X-rays (Shimojo et al., 1996; Shimojo and Shibata, 2000). Polar coronal jets were studied by Wang et al. (1998) using images of the LASCO (Large Angle and Spectrometric COronagraph) and EIT (Extreme ultraviolet Imaging Telescope) instruments on board SOHO (Solar and Heliospheric Observatory). Further observations were reported by Alexander and Fletcher (1999) with the TRACE (Transition Region And Coronal Explorer) spacecraft. More recently, also Hinode has provided important data on polar jet parameters (Savcheva et al., 2007; Kamio et al., 2007; Moreno-Insertis, Galsgaard, and Ugarte-Urra, 2008; Filippov, Golub, and Koutchmy, 2009). X-ray jets have typical lengths of $10^{4}-4 \times 10^{5} \mathrm{~km}$, widths of $5 \times 10^{3}-10^{5} \mathrm{~km}$, and speeds ranging from 
10 to $1000 \mathrm{~km} \mathrm{~s}^{-1}$ (Shimojo et al., 1996). It is usually assumed that the jet is the result of magnetic reconnection phenomena happening in the solar corona (Shibata et al., 1992; Yokoyama and Shibata, 1995, 1996; Moreno-Insertis, Galsgaard, and Ugarte-Urra, 2008; Pariat, Antiochios, and DeVore, 2009). Coronal jets can be observed both in active regions, quiet sun, and coronal holes (Shimojo et al., 1996). A polar coronal jet has been studied for the first time stereoscopically with observations from the STEREO/SECCHI imagers by Patsourakos et al. (2008), demonstrating the existence of helical magnetic field lines in this event.

The two points of view provided by the twin STEREO spacecraft are very helpful for the identification of coronal hole jets. Indeed, for the first time it is possible with the STEREO/SECCHI data to assess what the three dimensional (3D) structure of the jet is, to understand what projection effects are present in single point observations, and to try to establish the true 3D velocity for the jet (Patsourakos et al., 2008).

Here we present the first catalogue of STEREO polar coronal jets, comprising 79 events, which were observed by both EUVI imagers in the ultraviolet, and contemporarily by the COR1 coronagraph in white light, during the period at solar minimum at the end of solar cycle 23 from March 2007 to April 2008. This time interval, during which the separation angle between the two spacecraft increased from 2 to 48 degrees with respect to the Sun, includes two time periods of high time cadence observations in May 2007 and January 2008.

The criteria according to which the coronal jet events were identified and selected from the SECCHI dataset are explained in Section 2 of this paper, including a brief summary of their basic characteristics. Prototype events which also emphasize the difference of the viewpoints when seen either from STEREO A or from STEREO B are shown in Section 3. In Section 4, the lifetime distributions of jets in the different wavelengths in the EUVI and in COR1 FOV are discussed. The conclusions are given in Section 5, followed by the catalogue of polar coronal jets in the appendix.

\section{Selection Criteria and Statistics of the Jet Catalogue}

SECCHI images are taken at four EUV wavelengths centered at $171 \AA, 195 \AA, 284 \AA$ with a time cadence of $2.5,10,20$ minutes, and at $304 \AA$ with a time cadence of 10 minutes. During high time cadence observations on 5-19 May 2007, and on 7-20 January 2008 the time cadence was $75 \mathrm{~s}$ in the 171 channel. The pixel size of the EUVI CCD on the solar disk has a spatial resolution of $1.6 \mathrm{arc} \mathrm{sec}$. Because of the low time cadence of the $284 \AA$ observations, in this jet study more events are seen in the 171,195 and $304 \AA$ wavelength ranges. We began our systematic study of coronal jets in the SECCHI EUVI A and B data after STEREO's completed in-orbit insertion and the official science mission start on 22 January 2007, and after some follow on period of routine imaging, in March 2007. For the search of coronal jets EUVI images were used that are provided at the SECCHI website http://secchi.nrl.navy.mil/. SECCHI EUVI A and B daily movies are available at http://cdaw.gsfc.nasa.gov/stereo/daily_movies/. The existence of subsequent jets in the COR1 A and B FOVs were studied in the daily movies in intensity running difference available at http://cor1.gsfc.nasa.gov/dailymov/MPEG/. The running difference movies allow one to identify faint transient coronal structures, e.g., those hidden by coronal streamers.

In order to carry out a quantitative study of coronal jets, after identification of the events, SECCHI data have been analyzed with the SolarSoft package (http://www.Imsal.com/ solarsoft/), which provides routines for sophisticated SECCHI image processing. The data are processed with the secchi_prep routine and specific commands such as 

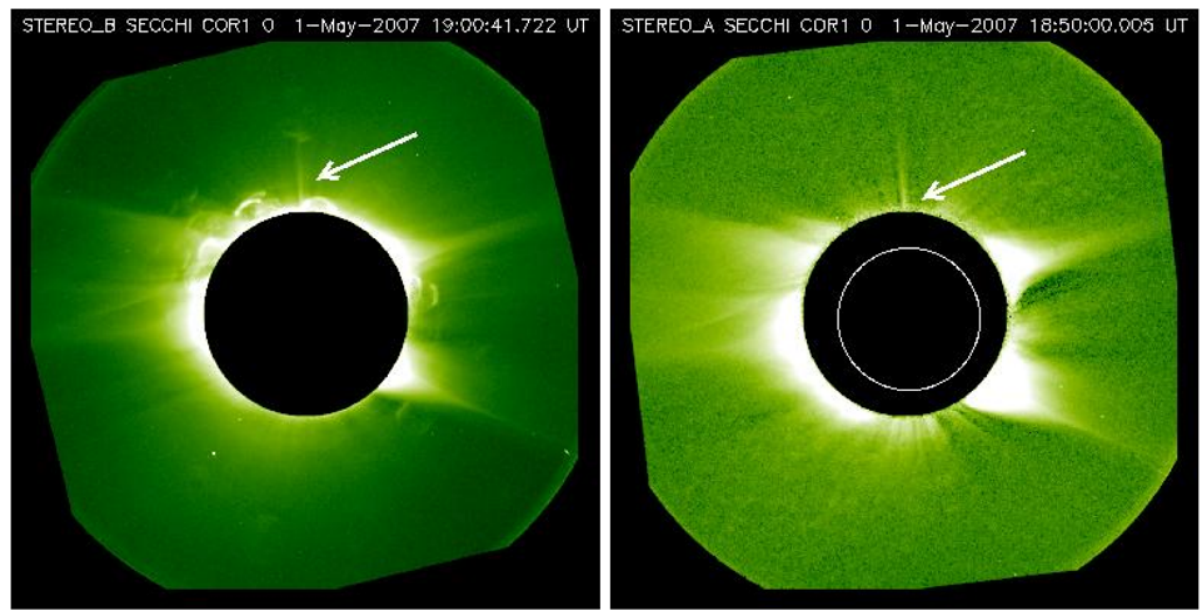

Figure 1 Coronal jet seen in the COR1 field of view for STEREO B (left) and A (right) on 01 May 2007 near the Sun's north pole (event no. 10 in the catalogue). Note the different offset of the STEREO A and B COR1 occulters with respect to the Sun's center due to different pointing. The spacecraft angular separation was $\Delta \phi_{\mathrm{AB}} \simeq 6.18^{\circ}$.

(plot_map, scc_triangulate, cursor) are used in order to obtain the values for the position angle of the events at the solar limb.

A selection criterion applied to the list of candidate jet events derived from the SECCHI images and COR difference movies, covering 256 events, was the unique visibility of the jets not just in the $171 \AA$ or $195 \AA$ observations of EUVI but also at least in either the COR1 A or COR1 B observations. This criterion ensures that a jet is not confined to the low coronal FOV of EUVI, i.e. to a height of $1.7 R_{\odot}$, and is not simply a spicule or macrospicule event (Wang et al., 1998; Yamauchi et al., 2004). The visibility of the jet in the $171 \AA$ or the $195 \AA$ lines implies that the observed jet is comprising hot plasma at typical temperatures of $\sim 10^{6} \mathrm{~K}$. The condition that jets be detected by both STEREO A and STEREO B EUV imagers reduces the number of events to 79 . Figure 1 shows an example of a typical coronal jet identified in the COR1 B and A fields of view.

In the catalogue in the appendix we provide information on the date for all of the identified 79 jet events, the angular separation $\Delta \phi_{\mathrm{AB}}$ between STEREO A and STEREO B as seen from the Sun at the time of the event, the time of observation with EUVI at the different wavelengths and with COR1, the position angle $\alpha$ and $\beta$, respectively in the EUVI and COR1 imagers at the solar limb, measured positive counterclockwise from the solar North and, when possible, the result of the assessment of the jet's morphology. The spatial distribution of jet positions corresponds to 45 events (57\%) found in north polar coronal holes (NPCHs) and to 34 events (43\%) in south polar coronal holes (SPCHs). The question whether the slight difference in the number of northern and southern events is related to a different areal size of the coronal holes in both solar hemispheres is beyond the scope of the present study. Another possibility for a north - south asymmetry is that the view angle of the polar regions for the two spacecraft was different.

A number of equatorial jets have also been found in the course of the search for coronal jet events, indicating that coronal jets are an overall coronal feature rather than limited to the polar regions, in agreement with the findings of Moreno-Insertis, Galsgaard, and UgarteUrra (2008). Figure 2 shows a sample event as a sequence of images from STEREO B at 


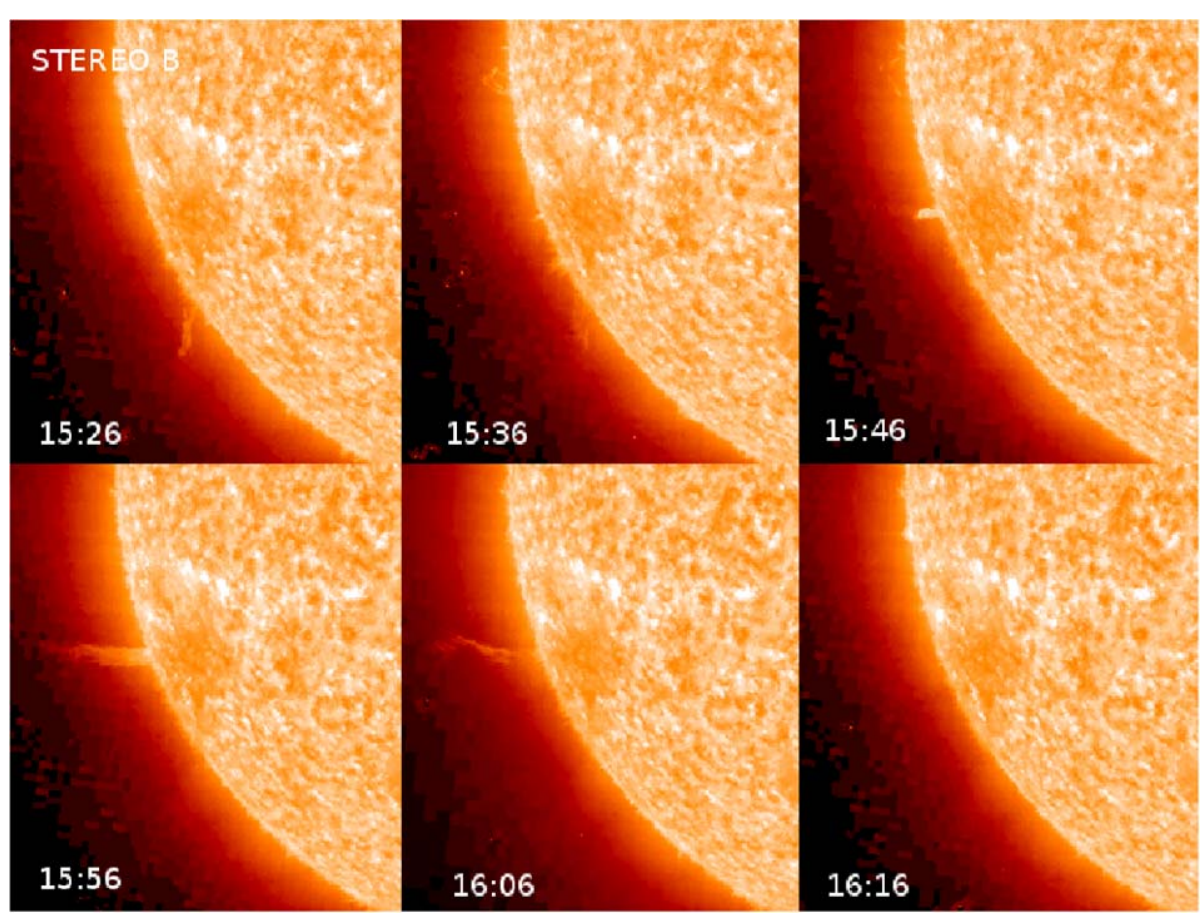

Figure 2 Sequence of images from STEREO B showing the evolution of an equatorial coronal jet on 11 November 2007.

$304 \AA$, in which the equatorial jet on 11 November 2007 has been identified. The corresponding equatorial coronal hole can be seen as a darker cooler area. The event was also visible in the $171 \AA$ and $195 \AA$ wavelengths. However, because of the usual presence of large-scale equatorial coronal structures, as is typical near the solar minimum, e.g., the presence of a streamer belt, only for very few equatorial jet events a clear subsequent signal could be identified in the COR1 data without improved image analysis. The equatorial jets identified so far from the SECCHI data are not included in the jet catalogue presented in the appendix, but will be the subject of further analysis.

\section{Typical Morphology of Coronal Jets}

Based on previous results, coronal jets can be basically classified into the following two categories (Table 1): i) Eiffel Tower (ET) jets which resemble a shape like small helmet streamers or an inverted-Y shape, and may correspond to the magnetic topology of a small-scale photospheric bipole reconnecting with ambient open unipolar field lines of opposite polarity at its loop tops (Shimojo et al., 1996; Yamauchi et al., 2004; Patsourakos et al., 2008; Pariat, Antiochios, and DeVore, 2009; Filippov, Golub, and Koutchmy, 2009); ii) $\lambda$-jets in which a small-scale photospheric magnetic bipole reconnects with ambient unipolar field lines near its footpoints (Shibata et al., 1992; Yokoyama and Shibata, 1996; Filippov, Golub, and Koutchmy, 2009). It should be noted that the EUVI observations only allow us to distinguish whether a jet occurred close to loop tops or the loop footpoints, i.e. we do not observe directly the magnetic field structure. An ideal case would thus be 
Table 1 Statistics of jet morphology. In the table we give the event type, the number of helical events over the total number for the specific type, the locations in which they seem to occur. Edge (Inside) indicates jets which are close to (detached from) the coronal hole boundary. In the last row are the total number of events that show helical features.

\begin{tabular}{llrrr}
\hline Classification & Number of events & Limb & Edge & Inside \\
\hline Eiffel Tower & $19 / 37$ & 21 & 2 & 14 \\
Lambda & $4 / 12$ & 4 & 5 & 3 \\
Micro-CME & $2 / 5$ & 4 & 1 & 4 \\
Not classified & $6 / 25$ & 17 & 4 & \\
Helical structure & 31 & & & \\
\hline
\end{tabular}

the phase of the STEREO mission when the viewing angle with respect to the Sun-Earth line had increased to about 90 degrees to correlate limb observations from STEREO with disk centered magnetograms from Hinode and SOHO. The morphology for each of the jet events listed in the table of the appendix was investigated for its morphological characteristics according to the above two categories. Figures 3 and 4 show archetype "Eiffel tower" and "lambda" events observed by SECCHI EUVI A and B. Figure 3 shows a sequence of images of a north polar coronal jet taken at $171 \AA$, seen by both STEREO A and STEREO B on 1 May 2007 (event no. 10 in the catalogue). The fast ejection of hot material, as well as the bright loop at the bottom of the jet, are clearly seen by both spacecraft. The angular separation was $\Delta \phi_{\mathrm{AB}} \simeq 6.18^{\circ}$, and the difference in the viewpoints is already becoming obvious and it is also possible to identify the helical structure of the ejection.

Figure 4 shows a sequence of images at $284 \AA$ for a north polar jet taken on 17 November 2007 , when both spacecraft were separated by $\Delta \phi_{\mathrm{AB}} \simeq 40.57^{\circ}$ (event no. 51). In STEREO EUVI A images, the jet is associated with the presence of a bright point at one leg of the loop and the ejection is developed at the opposite leg. In STEREO EUVI B only the presence of the bright point is visible. For this same event, images in $195 \AA$, separated in time of one minute with respect to images at $284 \AA$, show ejection at the same heliographic position but the footpoint seems to be characterized by a small loop (Figure 5). This event shows that the jet features, and even its very detection, can change with the point of view and with different wavebands. Moreover, images from STEREO A clearly show a shift from left to right in the jet position. Such a shift suggests an evolution of the magnetic reconnecting region, maybe a "kink" instability, which could be compared with the results of numerical simulations (e.g. Yokoyama and Shibata, 1996; Moreno-Insertis, Galsgaard, and Ugarte-Urra, 2008; Pariat, Antiochios, and DeVore, 2009) and other data analyses (e.g. Savcheva et al., 2007; Filippov, Golub, and Koutchmy, 2009). As can be seen from Figures 3 and 4, the jet footpoints typically appear as low coronal bright points or loops. These features sometimes seem to coexist, whereas in other cases they appear from different perspectives as the same structure in the SECCHI A and B images. We interpret this finding as projection effects of small loops resembling coronal bright points at lower coronal altitudes when viewed edge on. These observations emphasize the importance of different perspectives.

Another important feature of the jets, which we investigated, was whether a helical structure along the jet axis, as first reported by Patsourakos et al. (2008), could be identified. A sample event is presented in Figure 6. Out of the total number of 79 events, 31 events clearly revealed a helical structure, as indicated in the table of the jet events in the appendix. Figure 6 shows a north polar jet which also exhibited a prominent helical structure but not seen to originate from an emerging closed loop system in 171/195 $\AA$. 

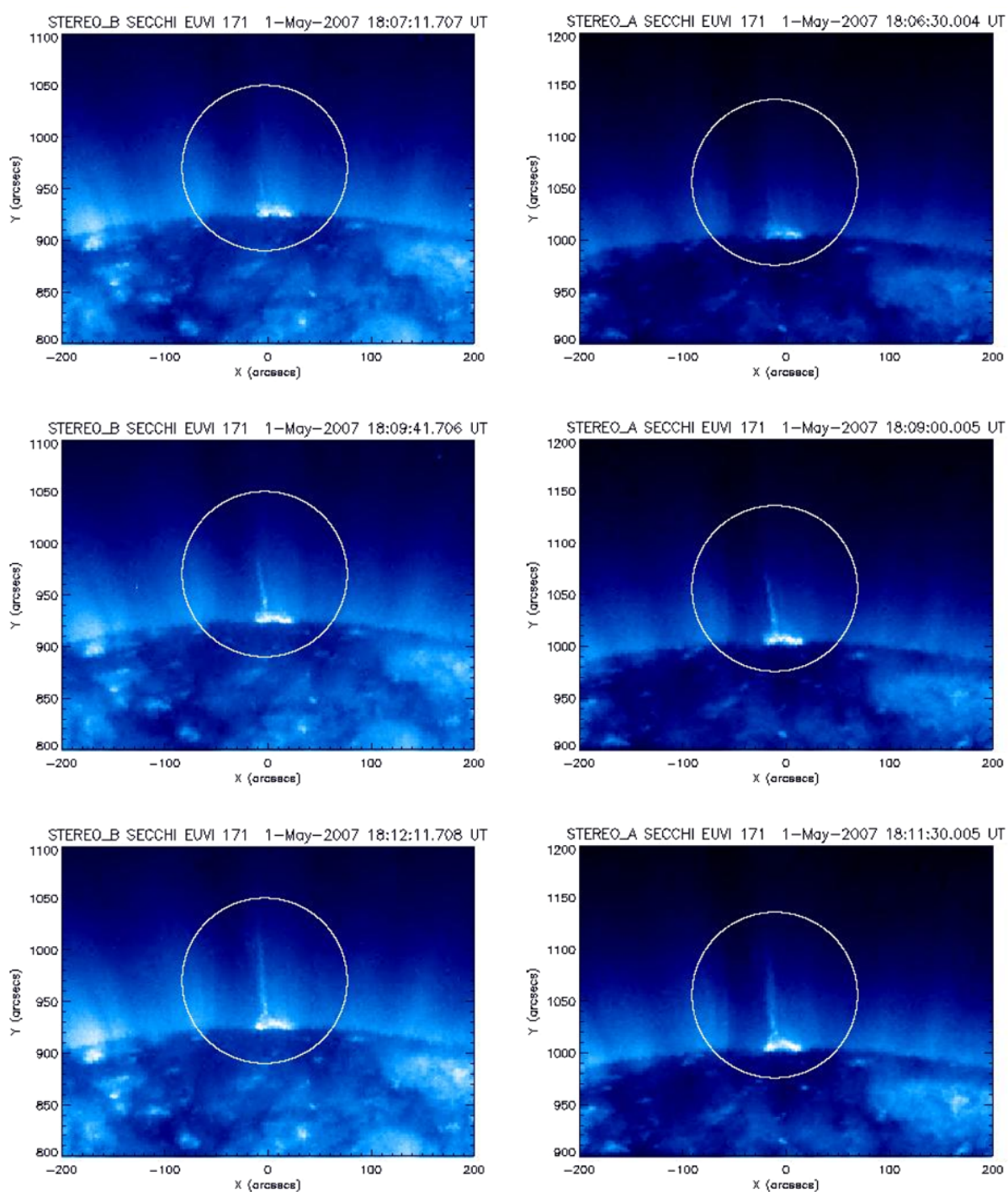

Figure 3 Sample "Eiffel Tower" event in the north polar coronal hole on 01 May 2007 imaged at $171 \AA$. Left: view from STEREO/SECCHI EUVI B. Right: view from STEREO/SECCHI EUVI A. Note that in this case the small loop below the jet is clearly resolved. Same events as in Figure 1.

It was observed at $304 \AA$ on 8 February 2008 (event no. 75), when the angular separation was $\Delta \phi_{\mathrm{AB}}=45.45^{\circ}$. It can be seen that the two helical arms appear well separated in STEREO B, while they partly overlap in STEREO A. This helical structure is also clearly seen at $171 \AA$ and $195 \AA$, although the images are less bright. Since the presence of a helical structure is important to test the validity of different jet models $(e . g$. Pariat, Antiochios, and DeVore, 2009) these events are important cases for further modeling. The question of why all jets do not show the helical structure cannot be uniquely answered. A possibility is that they were very narrow so that the twist could not be re- 

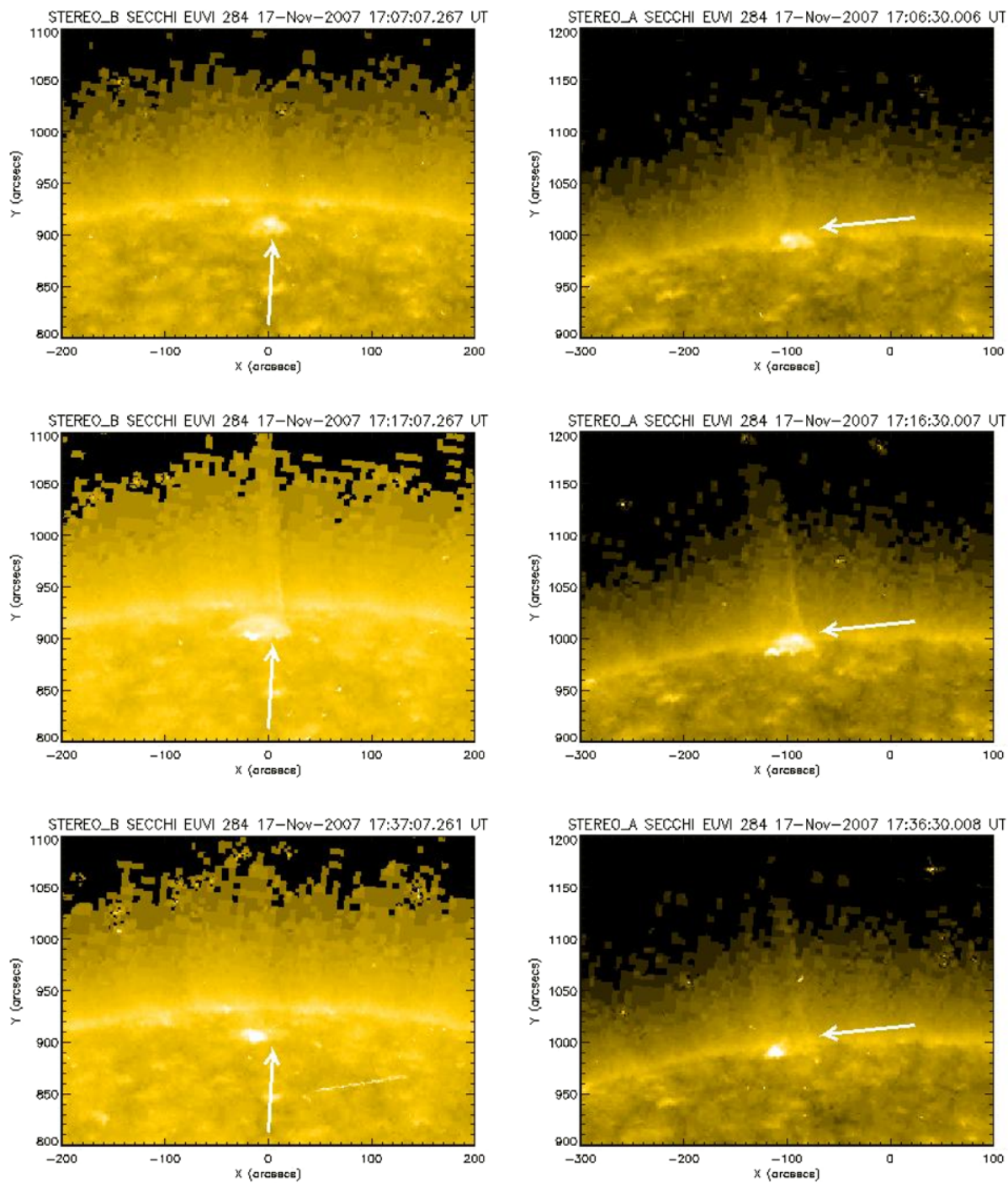

Figure 4 Sample " $\lambda$ " event in the north polar coronal hole on 17 November 2007 as observed by SECCHI EUVI A and B at $284 \AA$. Spacecraft angular separation: $\Delta \phi_{\mathrm{AB}} \simeq 40.57^{\circ}$. Left: view from STEREO B. Right: view from STEREO A.

solved, as supported by the cases when the jet widths were similar from both perspectives (e.g. events no. 20, 21, 29, 67, 73). This is what one expects for jets that, to first order, are azimuthally-symmetric structures, i.e. invariant by rotation, so that they show the same width from different viewpoints. On the contrary, a slab-like geometry would lead to significantly different widths for different perspectives. A detailed study of these characteristics would provide further insight into the validity of 3D models invoking magnetic structures, which may be axially symmetric or not (Moreno-Insertis, Galsgaard, and Ugarte-Urra, 2008; Pariat, Antiochios, and DeVore, 2009). Another possibility could be that the helical phase is so transient that observations at every few minutes are not sufficient to catch it. 

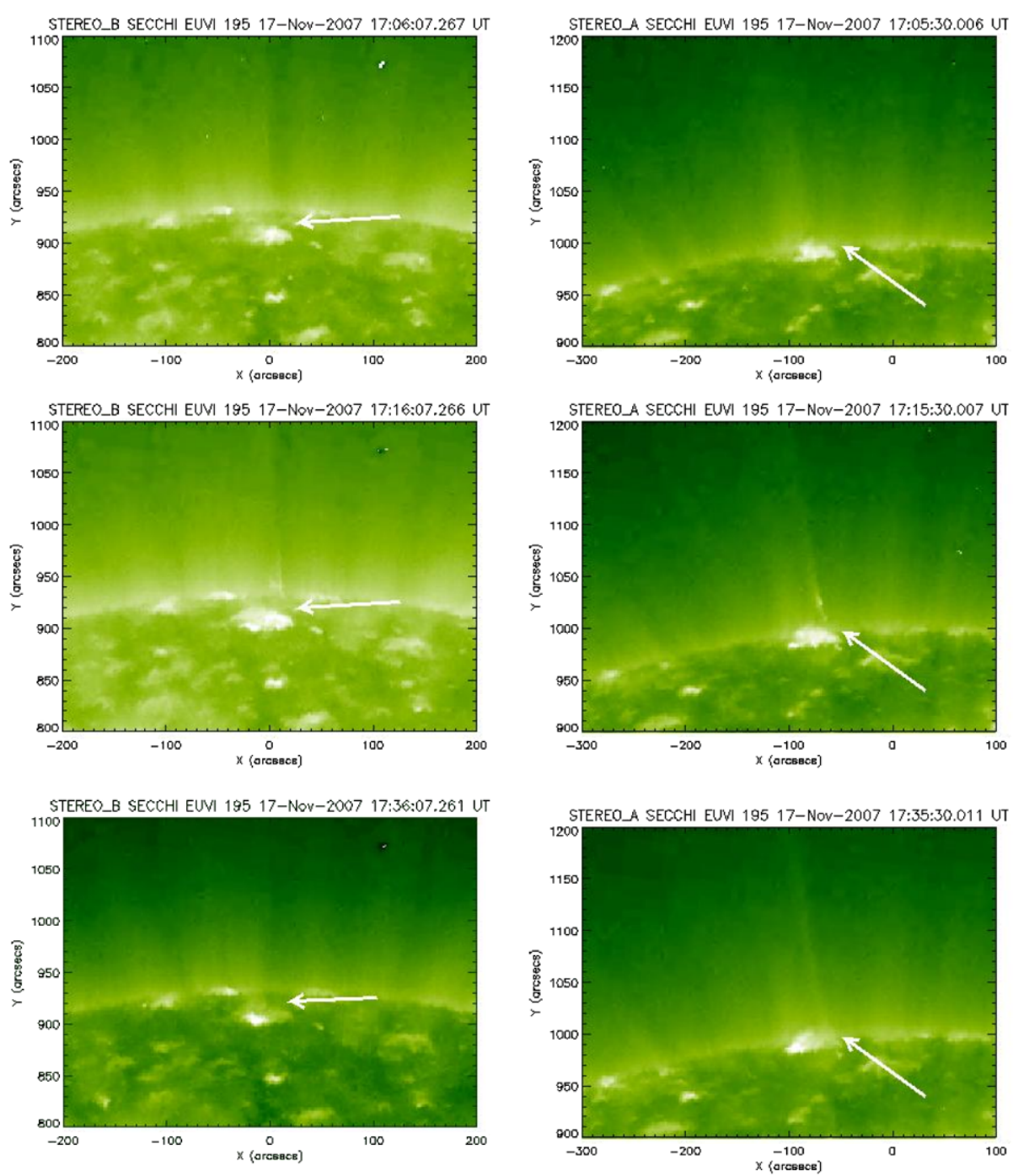

Figure 5 Same " $\lambda$ "-type coronal jet event as in Figure 4, but seen at $195 \AA$ by STEREO B (left) and A (right) on 17 November 2007.

A surprise during the study of the jet morphologies was to find events that revealed the same morphology as typically large-scale three part structured CMEs, consisting of a bright leading edge, a dark void and bright trailing edge (being the prominence material) but on much smaller scales (Cremades and Bothmer, 2004). These so-called micro-CME events are described in more detail in the paper by Bothmer and Nisticò (in preparation). Another sample jet event (no. 41 of the catalogue) showing the appearance of a twisted small-scale prominence at $304 \AA$ arising from inside the south coronal hole on 12 October 2007 is presented in Figure 7. Such twisted prominences are well observed features on a much larger scale (http://sohowww.nascom.nasa.gov/gallery/bestofsoho.html). The spacecraft angular separation at this time was $\Delta \phi_{\mathrm{AB}} \simeq 35.84^{\circ}$. The difference in the viewpoints is 

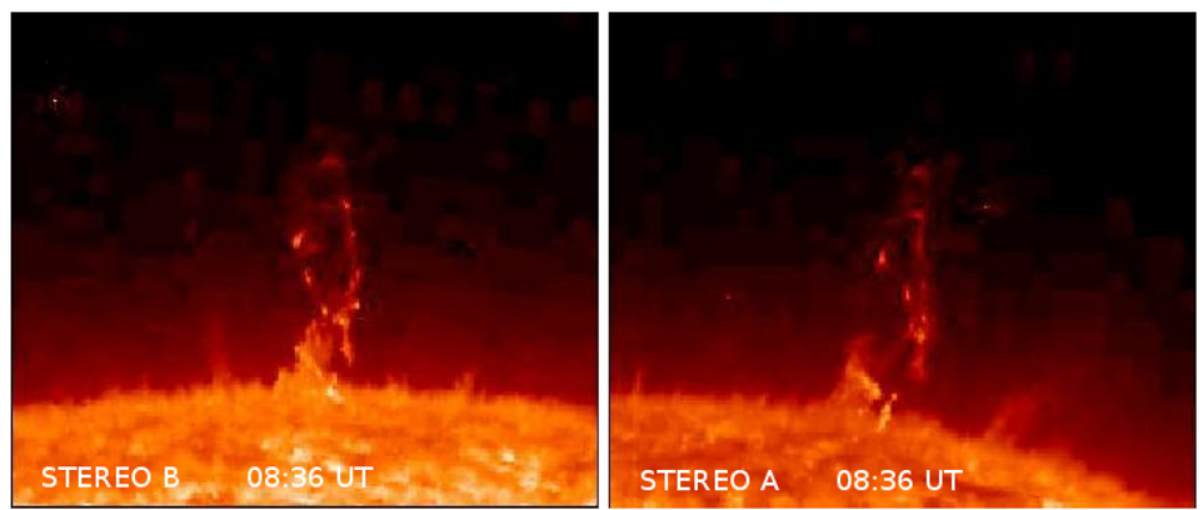

Figure 6 The helical jet event on 08 February 2008. Spacecraft angular separation: $\Delta \phi_{\mathrm{AB}}=45.45^{\circ}$
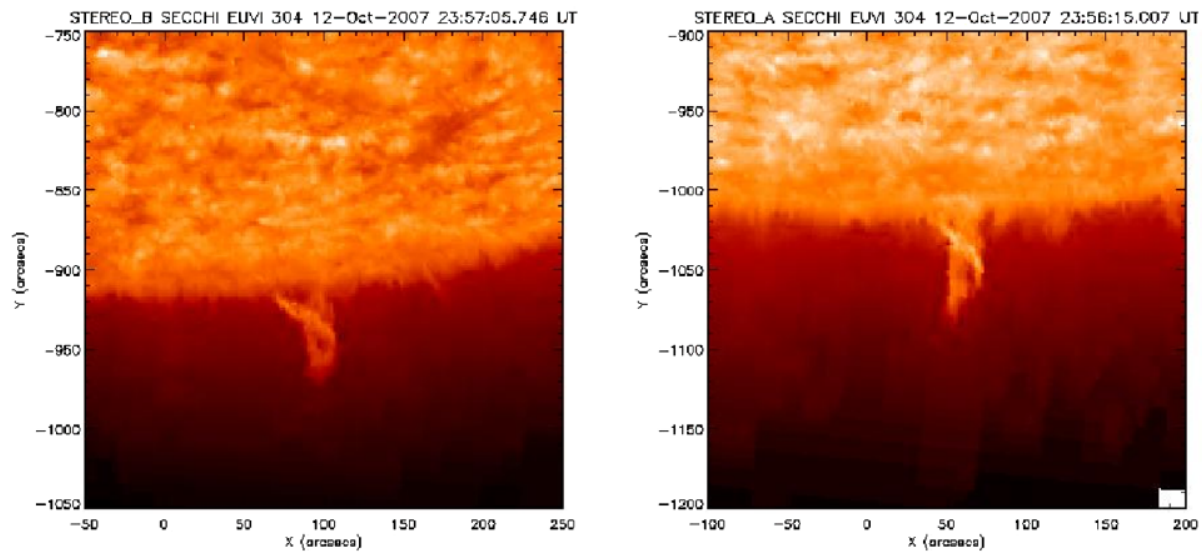

Figure 7 Images of twisted prominence material in the trailing portion of a jet observed on 12 October 2007. Spacecraft angular separation: $\Delta \phi_{\mathrm{AB}} \simeq 35.84^{\circ}$.

obvious and confirms that it is a twisted 3D loop indeed. It seems likely that the overall magnetic topology depends on the source region characteristics of the underlying photospheric magnetic field, as in the case of large-scale CMEs found to arise from bipolar magnetic field regions on larger scale as shown by Cremades and Bothmer (2004). Similar to the largescale CMEs, the micro-CMEs may also evolve from bipoles with enhanced magnetic flux compared to the surrounding fields but on much smaller scales. This is similar to the observation that coronal jets seem to arise close to small bipoles within the coronal holes as shown by Shimojo et al. (1996). However it is beyond the scope of this study to fully investigate the coupling of the coronal and photospheric structures. Overall we found evidence for 5 micro-CMEs in the total set of 79 events.

The list of coronal jet events presented in the appendix provides information on the identified morphology of the individual events. However, it should be pointed out that for a number of events a unique classification was not possible, because sometimes the jet features were different in each wavelength and sequences at different time cadences had to be investigated; in the appendix this is indicated by a question mark. 

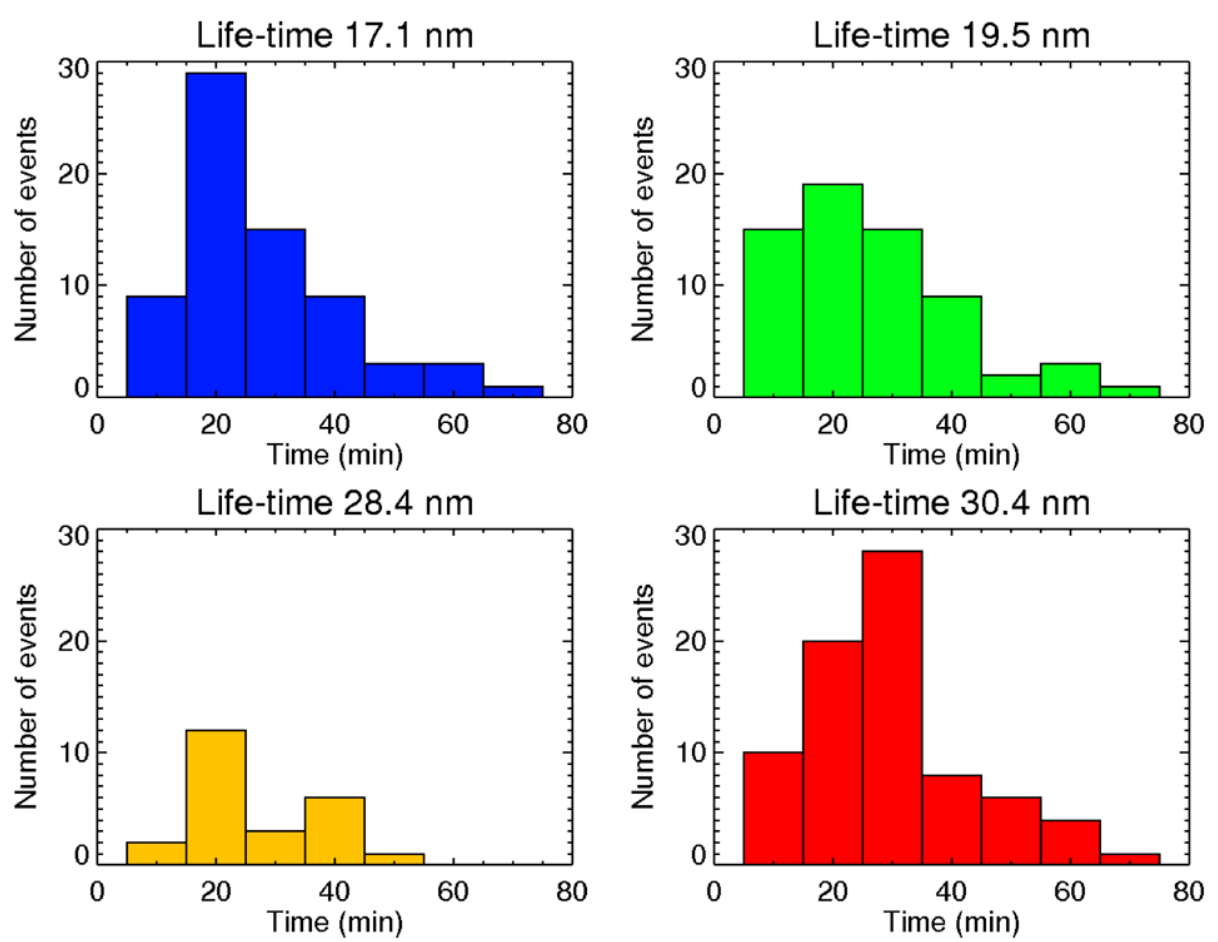

Figure 8 Distribution of the lifetime of coronal jets seen by STEREO/SECCHI at 171, 195, 284 and $304 \AA$. Note the shift of the peak in the lifetime distribution at $304 \AA$.

\section{Coronal Jet Lifetimes}

Inspection of the polar jets reported in the catalogue also allows one to obtain some basic information about the duration of observation in the EUVI field of view, i.e., about the lifetime of the jets at the different wavelengths in the low corona. These EUVI visibilities correspond to the lifetimes given in the third column of the catalogue for each wavelength. A statistical distribution for the lifetimes of all jets at the different wavelengths between $1-1.7 R_{\odot}$ in bins of 10 minutes is presented in Figure 8 .

It should be noted that the estimated lifetime is influenced by the different time cadence with which the EUVI telescope operates: usually a time cadence of $2.5 \mathrm{~min}$ is used for $171 \AA$, while the cadence is $10 \mathrm{~min}$ for $195 \AA$ and $304 \AA$. It can be seen from Figure 8 that the lifetimes of the jets analyzed in this study at $171 \AA$ and $195 \AA$ are peaked at 20 min; conversely, at $304 \AA$, the lifetime distribution is peaked at $30 \mathrm{~min}$. The lifetime distribution at $284 \AA$ is peaked at $20 \mathrm{~min}$ and $40 \mathrm{~min}$ but the number of events is lower than in the other wavebands; in this case the two peaks may just correspond to multiples of the most used cadence. The difference in lifetimes emphasizes the fact that looking at different wavelengths implies observing the solar corona at different temperatures: the spectral line at $304 \AA$ corresponds to lower temperatures $(\sim 80000 \mathrm{~K})$, which suggests that the duration of the event is usually longer at lower temperatures. On the other hand, the shorter lifetime at $171 / 195 \AA$ could also be related to the fact that at these wavelengths jets have coronal temperatures $\left(\sim 10^{6} \mathrm{~K}\right)$ and dim very fast with distance, since they travel in the hot corona and contrast is low. Conversely, $304 \AA$ jets have larger contrast since there is not so strong 

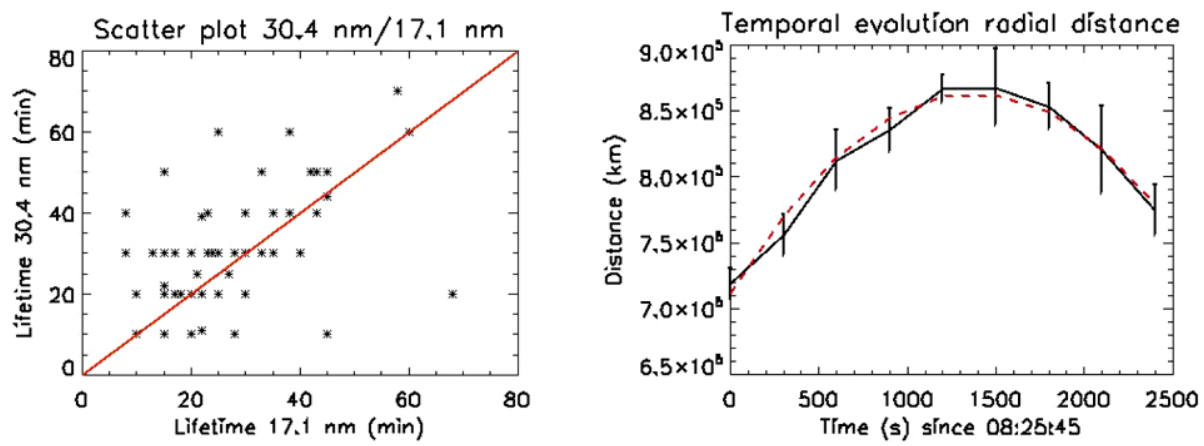

Figure 9 Left: scatter plot of $304 \AA$ and $171 \AA$ lifetimes. The majority of events show longer duration in $304 \AA$ than in $171 \AA$. Right: a plot of the leading edge position versus time for the micro-CME of 6 May 2007, at $304 \AA$. We can obtain the starting speed of leading edge jet $\left(v_{0}=(219.86 \pm 22.38) \mathrm{km} \mathrm{s}^{-1}\right)$ and the acceleration $\left(a=(-0.1596 \pm 0.0198) \mathrm{km} \mathrm{s}^{-2}\right)$ by a quadratic fit (red dashed line).

emission at this wavelength in the corona. This implies that the lifetimes at $195 \AA$ could be longer by at least one exposure. From Figure 9(a) it can be seen that for each event the lifetime is usually shorter at 171 than $304 \AA$; this can be compared with the lifetime in the $\mathrm{X}$-rays, which is even shorter, as reported by Savcheva et al. (2007) and Cirtain et al. (2007). A very rough estimate of jet speeds can be obtained from the jet lifetime by assuming that the jet speed does not change too much during the transit time in the EUVI field of view of $0.7 R_{\odot}$ beyond the limb. This means that $171 \AA$ plasma has an estimated outward propagating speed of about $400 \mathrm{~km} \mathrm{~s}^{-1}$, whereas $304 \AA$ cooler material is moving at lower speeds of about $270 \mathrm{~km} \mathrm{~s}^{-1}$. If we accept these speed estimates as typical of the corresponding jets, we can infer that the $304 \AA$ cooler material is not capable to reach a speed sufficient to leave the Sun at the given height, so that material is falling back (similar to spicula matter), as can be noted in some events (e.g. no. 14, 15, 71, 73) (the escape speed from the Sun at the distance of $1.7 R_{\odot}$ is $\sim 360 \mathrm{~km} \mathrm{~s}^{-1}$ ). Similar results, based on Hinode jet observations, were reported by Culhane et al. (2007).

A more definitive way to measure the jet speed is by determining the leading edge jet position in 3D for a number of consecutive frames. This has been done with the help of the SolarSoft routine (scC_triangulate) and is shown for the event no. 15 of the catalogue in Figure 9(b). A parabolic fit yields a maximum jet speed of $\sim 200 \mathrm{~km} \mathrm{~s}^{-1}$, consistent with the above estimates at $304 \AA$. Also, the inferred (sunward) acceleration is less than the solar gravity, which shows that electromagnetic forces are still acting on the jet material. Another 3D velocity determination will be presented elsewhere. A similar analysis was carried out by Gilbert et al. (2000), using Mauna Loa Solar Observatory $\mathrm{H} \alpha$ observations of projected radial positions and velocities of active and eruptive prominences. In those cases both negative and positive radial accelerations were found, highlighting the importance of the magnetic topology for the plasma dynamics.

An estimate of the lifetimes can also be obtained from the coronagraph observations. In COR1 A the peak of the distribution is centered near $70 \mathrm{~min}$, while in COR1 B the peak of the distribution is centered near $80 \mathrm{~min}$. A lifetime of 75 minutes for the field of view of COR 1 corresponds to a speed of about $390 \mathrm{~km} \mathrm{~s}^{-1}$, which is in good agreement with the speed derived for the hot $171 \AA$ plasma components of the jets. The fact that the estimated lifetime in COR1 B is higher by ten minutes might be explained by its better stray-light rejection, since this feature allows one to trace jets farther away. 


\section{Conclusions}

In this work we presented the first comprehensive statistical study of polar coronal jets with the SECCHI instrument package on the STEREO spacecraft, using EUVI A and B and COR1 A and B data. A catalogue of 79 jets identified from simultaneous EUVI and COR1 coronagraph observations by both spacecraft has been compiled. The observations were taken during a period at solar activity minimum from March 2007 to April 2008. From a systematic investigation of the 79 events observed by both spacecraft at separation angles between 2 and 48 degrees we find the following basic properties of coronal jets.

- 37 Eiffel tower-type jets in which jets show a shape similar to an inverted-Y and they are associated to the magnetic configuration of a small magnetic bipole reconnecting with the ambient coronal field at its loop top.

- 12 lambda-type jets in which ejection is observed to be shifted from the position of a bright point or a small loop, and this topology is associated to the magnetic configuration of a small bipole reconnecting with the unipolar coronal field at its footpoint.

- Besides the previous two morphologies, there are five micro-CME-type events (Bothmer and Nisticò, in preparation) characterized by the evidence of a small loop that elongates from the solar surface and resemble the CMEs but on smaller scale.

Without more sophisticated analyses of jets it is difficult to provide a reliable interpretation why we have identified about three times more ET than $\lambda$ events. ET events at loop tops likely have better visibility against the background compared to reconnection processes happening at the footpoints because those appear bright and heating processes are less visible. Another plausible interpretation would be that ET-type jets could require some build-up of magnetic energy and then an instability like the one discussed by Patsourakos et al. (2008), Pariat, Antiochios, and DeVore (2009). Therefore, whenever they occur, more energy has been stored in them and therefore released by them and the jets are brighter, and thus more detectable. On the other hand, $\lambda$ jets can occur more "easily" without so much energy having been stored, and therefore are less observable, even though they may be more numerous.

Overall there are 31 events that clearly exhibit the helical structure of the magnetic field. It is important to note that these findings imply that a jet observed by a coronagraph at heights of $\sim 1.5 R_{\odot}$ can have different low coronal onset scenarios in terms of the magnetic fields structure and its evolution.

The typical lifetimes in the EUVI FOV are 20 minutes at $171 \AA$ and 30 minutes at $304 \AA$, while in COR1 the lifetimes are peaked at around 70-80 minutes. The corresponding estimated speeds with respect to the FOV of the EUVI and COR1 telescopes are $400 \mathrm{~km} \mathrm{~s}^{-1}$ for the hot $171 \AA$ plasma component and only $270 \mathrm{~km} \mathrm{~s}^{-1}$ for the cooler $304 \AA$ chromospheric component. The speed of $400 \mathrm{~km} \mathrm{~s}^{-1}$ is comparable to that derived from the COR1 FOV of $390 \mathrm{~km} \mathrm{~s}^{-1}$.

The present catalogue can serve several purposes. One is to undertake a more detailed study of specific events to determine the $3 \mathrm{D}$ velocity and acceleration and to carry out the $3 \mathrm{D}$ reconstruction of the jet, to compare with numerical simulations. A second purpose is to look for the association between jets and other chromospheric phenomena, like spiculae, plumes, etc., (Raouafi et al., 2008) which is useful for constraining more complete physical models of the solar activity in the coronal holes. A third purpose is to fill the gap between large scale coronal phenomena like CME and the small-scale phenomena in polar coronal holes. 
Acknowledgements It is a pleasure to thank all the STEREO staff, without which this work would not have been possible. G.N. acknowledges support from an Erasmus grant during his stay in Goettingen. V.B. acknowledges the support of the project Stereo/Corona by the German Bundesministerium für Bildung und Forschung through the deutsche Zentrum für Luft- und Raumfahrt e.V. (DLR, German Space Agency) as a collaborative effort with the Max-Planck-Institut für Sonnensystemforschung (MPS) under grant 50 0C 0904. Stereo/Corona is a science and hardware contribution to the optical image package SECCHI, developed for the NASA STEREO mission launched in 2006. The SECCHI data used here were produced by an international consortium of the Naval Research Laboratory (USA), Lockheed Martin Solar and Astrophysics Lab (USA), NASA Goddard Space Flight Center (USA), Rutherford Appleton Laboratory (UK), University of Birmingham (UK), Max-Planck-Institut for Solar System Research (Germany), Centre Spatiale de Liège (Belgium), Institut d'Optique Théorique et Appliquée (France), and Institut d'Astrophysique Spatiale (France). G.Z. was supported in part by the Italian INAF and by the Italian Space Agency, contract ASI n. I/015/07/0 "Esplorazione del Sistema Solare". We would like to thank the referee for his/her careful and constructive criticism, which has allowed us to substantially improve the paper.

Open Access This article is distributed under the terms of the Creative Commons Attribution Noncommercial License which permits any noncommercial use, distribution, and reproduction in any medium, provided the original author(s) and source are credited.

\section{Appendix: Catalogue of Polar Coronal Hole Jets}

In this appendix, polar jet events are organized in a catalogue. Each event is labeled by progressive number, date of observation, angular separation $\Delta \phi_{\mathrm{AB}}$ between STEREO A and STEREO B, time of visibility in EUVI and in COR1, position angle in EUVI $(\alpha)$ and in COR1 $(\beta)$, brief indication on the morphology of the event and presence of helicity. The position angles are given for STEREO A, except when differently stated. The following abbreviations are used: ET: Eiffel tower; Lambda: lambda jet; N(S)PCH: North (South) polar coronal hole; L: limb; I: inside; EDGE: edge of coronal hole; Hel: evidence of helical structure. The question mark ? indicates unclassified jets events.

\begin{tabular}{|c|c|c|c|c|}
\hline $\mathrm{N}^{\circ}$ & $\begin{array}{l}\text { Date } \\
\Delta \phi_{\mathrm{AB}}\end{array}$ & EUVI & COR1 & $\begin{array}{l}\text { Position } \\
\text { Morphology }\end{array}$ \\
\hline \multirow[t]{4}{*}{1} & 2007-03-19 & 171 16:08-16:38 & A: $16: 20-17: 30$ & SPCH, L \\
\hline & & 195 16:01-16:31 & B: $16: 20-17: 20$ & $\alpha(A)=164^{\circ}$ \\
\hline & $2.16^{\circ}$ & 284 16:08-16:38 & & $\beta(A)=158^{\circ}$ \\
\hline & & 304 16:01-16:31 & & $?$ \\
\hline \multirow[t]{4}{*}{2} & 2007-03-19 & 171 16:08-16:28 & A: $16: 10-17: 20$ & $\mathrm{SPCH}, \mathrm{L}$ \\
\hline & & $19516: 01-16: 31$ & B: $16: 20-17: 30$ & $\alpha(A)=176^{\circ}$ \\
\hline & $2.16^{\circ}$ & $28415: 58-16: 28$ & & $\beta(A)=175^{\circ}$ \\
\hline & & 304 & & $?$ \\
\hline \multirow[t]{4}{*}{3} & $2007-03-20$ & 171 & A: & NPCH, EDGE \\
\hline & & 195 & B: $08: 51-09: 31$ & $\alpha(B)=6^{\circ}$ \\
\hline & $2.20^{\circ}$ & 284 & & $\beta(B)=6^{\circ}$ \\
\hline & & 304 08:31-09:01 & & Lambda \\
\hline \multirow[t]{4}{*}{4} & 2007-03-24 & $17100: 38-00: 58$ & A: $01: 10-02: 20$ & $\mathrm{SPCH}, \mathrm{L}$ \\
\hline & & 195 00:31-00:51 & B: $01: 10-02: 30$ & $\alpha(A)=181^{\circ}$ \\
\hline & $2.44^{\circ}$ & 284 00:48 - 00:58 & & $\beta(A)=182^{\circ}$ \\
\hline & & 304 00:51 (very faint) & & ET \\
\hline
\end{tabular}




\begin{tabular}{|c|c|c|c|c|}
\hline $\mathrm{N}^{\circ}$ & $\begin{array}{l}\text { Date } \\
\Delta \phi_{\mathrm{AB}}\end{array}$ & EUVI & COR1 & $\begin{array}{l}\text { Position } \\
\text { Morphology }\end{array}$ \\
\hline \multirow[t]{4}{*}{5} & 2007-03-27 & 171 not very clear & A: $22: 10-23: 50$ & $\mathrm{SPCH}, \mathrm{L}$ \\
\hline & & 195 21:41-22:01? & B: $22: 10-23: 30 ?$ & $\alpha(A)=176^{\circ}$ \\
\hline & $2.72^{\circ}$ & $28421: 58-22: 08$ & & $\beta(A)=167^{\circ}$ \\
\hline & & $30421: 51-22: 11$ & & $?$ \\
\hline \multirow[t]{4}{*}{6} & $2007-03-30$ & 171 14:49-15:01 & A: $15: 20-16: 30$ & $\mathrm{NPCH}, \mathrm{I}$ \\
\hline & & $19514: 42-14: 52$ & B: $15: 10-16: 30$ & $\alpha(A)=1^{\circ}$ \\
\hline & $2.92^{\circ}$ & $28414: 40-15: 00$ & & $\beta(A)=2^{\circ}$ \\
\hline & & $30414: 51$ & & ET \\
\hline \multirow[t]{4}{*}{7} & 2007-04-01 & $17114: 24-14: 54$ & A: $14: 40-16: 00 ?$ & $\mathrm{SPCH}, \mathrm{L}$ \\
\hline & & $19514: 22-14: 52$ & B: $14: 50-15: 30 ?$ & $\alpha(A)=170^{\circ}$ \\
\hline & $3.08^{\circ}$ & 284 & & $\beta(A)=166^{\circ}$ \\
\hline & & 304 14:31-14:51 & & Lambda \\
\hline \multirow[t]{4}{*}{8} & 2007-04-08 & 171 15:09-15:31 & A: $15: 30-16: 30$ & SPCH, I \\
\hline & & $19515: 12-15: 22$ & B: $15: 40-16: 50$ & $\alpha(A)=192^{\circ}$ \\
\hline & $3.68^{\circ}$ & 284 & & $\beta(A)=200^{\circ}$ \\
\hline & & 304 15:11-15:22 & & Lambda \\
\hline \multirow[t]{4}{*}{9} & 2007-05-01 & $17111: 19-11: 34$ & A: $11: 20-12: 20$ & $\mathrm{SPCH}, \mathrm{L}$ \\
\hline & & $19511: 22-11: 42$ & B: $11: 30-12: 15$ & $\alpha(A)=183^{\circ}$ \\
\hline & $6.15^{\circ}$ & 284 & & $\beta(A)=186^{\circ}$ \\
\hline & & 304 11:11-11:41 & & $?$ \\
\hline \multirow[t]{4}{*}{10} & 2007-05-01 & 171 18:04-18:29 & A: $18: 30-20: 00$ & $\mathrm{NPCH}, \mathrm{L}$ \\
\hline & & 195 18:12-18:32 & B: $18: 20-20: 00 ?$ & $\alpha(A)=0^{\circ}$ \\
\hline & $6.18^{\circ}$ & $28418: 20$ & & $\beta(A)=1^{\circ}$ \\
\hline & & 304 18:11-18:31 & & ET-Hel \\
\hline \multirow[t]{4}{*}{11} & 2007-05-02 & 171 13:01-13:16 & A: $13: 10-14: 30$ & $\mathrm{SPCH}, \mathrm{EDGE}$ \\
\hline & & 195 13:02-13:22 & B: $13: 15-?$ & $\alpha(A)=153^{\circ}$ \\
\hline & $6.28^{\circ}$ & 284 13:00 & & $\beta(A)=152^{\circ}$ \\
\hline & & 304 13:01-13:51 & & ET-Hel \\
\hline \multirow[t]{4}{*}{12} & 2007-05-02 & $17120: 59-21: 21$ & A: $21: 20-22: 10$ & $\mathrm{NPCH}, \mathrm{L}$ \\
\hline & & $19521: 02-21: 22$ & B: $21: 10-22: 35$ & $\alpha(A)=0^{\circ}$ \\
\hline & $6.33^{\circ}$ & 284 & & $\beta(A)=1^{\circ}$ \\
\hline & & 304 21:01-21:31 & & ET-Hel \\
\hline \multirow[t]{4}{*}{13} & 2007-05-04 & 171 09:48 - 10:01 & A: & $\mathrm{SPCH}, \mathrm{L}$ \\
\hline & & 195 09:46-10:01 & B: & $\alpha(A)=177^{\circ}$ \\
\hline & $6.52^{\circ}$ & 284 09:46-10:01 & (diff.movie(A):10:15) & $\beta(A)=175^{\circ}$ \\
\hline & & 304 09:50-10:20 & & Lambda \\
\hline \multirow[t]{4}{*}{14} & 2007-05-05 & $17104: 03-04: 18$ & A: & $\mathrm{NPCH}, \mathrm{L}$ \\
\hline & & 195 04:06-04:21 & B: & $\alpha(B)=16^{\circ}$ \\
\hline & $6.62^{\circ}$ & 284 04:06 & (diff.movie(B):04:25 - & $\beta(B)=26^{\circ}$ \\
\hline & & 304 04:05-04:35 & $05: 05)$ & Micro-CME \\
\hline \multirow[t]{4}{*}{15} & 2007-05-06 & 171 08:26-08:48 & A: $08: 40-09: 15$ & SPCH, L \\
\hline & & 195 08:26-08:56 & B: $08: 55-09: 40$ & $\alpha(A)=172^{\circ}$ \\
\hline & $6.78^{\circ}$ & $28408: 26-08: 56$ & & $\beta(A)=167^{\circ}$ \\
\hline & & 304 08:31-09:10 & & Micro-CME \\
\hline
\end{tabular}




\begin{tabular}{|c|c|c|c|c|}
\hline $\mathrm{N}^{\circ}$ & $\begin{array}{l}\text { Date } \\
\Delta \phi_{\mathrm{AB}}\end{array}$ & EUVI & COR1 & $\begin{array}{l}\text { Position } \\
\text { Morphology }\end{array}$ \\
\hline 16 & 2007-05-07 & $\begin{array}{l}171 \\
19514: 46 \\
284 \\
30414: 50-15: 05\end{array}$ & $\begin{array}{l}\text { A: } 15: 30-16: 30 \\
\text { B:difficult } \\
\text { better seen } \\
\text { in diff.movie }\end{array}$ & $\begin{array}{l}\text { SPCH, L } \\
\alpha(A)=176^{\circ} \\
\beta(A)=177^{\circ} \\
?\end{array}$ \\
\hline 17 & 2007-05-09 & $\begin{array}{l}171 \text { 10:40 } \\
195 \text { 10:41 } \\
284 \text { 10:41 } \\
304 \text { 10:35-11:06 }\end{array}$ & $\begin{array}{l}\text { A: } 10: 50-11: 50 ? \\
\text { B: } 10: 40-11: 50 ?\end{array}$ & $\begin{array}{l}\text { NPCH, W, L } \\
\alpha(A)=346^{\circ} \\
\beta(A)=316^{\circ} \\
?\end{array}$ \\
\hline 18 & 2007-05-10 & $\begin{array}{l}171 \\
195 \\
284 \\
30407: 33-07: 51\end{array}$ & $\begin{array}{l}\text { A: } 08: 10-09: 30 \\
\text { B: }\end{array}$ & $\begin{array}{l}\text { SPCH, I } \\
\alpha(A)=175^{\circ} \\
\beta(A)=187^{\circ} \\
?\end{array}$ \\
\hline 19 & $7.50^{\circ}$ & $\begin{array}{l}171 \quad 17: 04-17: 31 \\
19517: 06-17: 26 \\
28417: 11-17: 26 \\
30417: 10-17: 35\end{array}$ & $\begin{array}{l}\text { A: } \\
\text { B: } \\
\text { (diff.movie(A):17:45) }\end{array}$ & $\begin{array}{l}\text { NPCH, L/EDGE } \\
\alpha(A)=346^{\circ} \\
\beta(A)=333^{\circ} \\
?\end{array}$ \\
\hline 20 & $9.38^{\circ}$ & $\begin{array}{l}17109: 28-09: 46 \\
19509: 25-09: 55 \\
284 \\
30409: 36-09: 56\end{array}$ & $\begin{array}{l}\text { A: 09:55-10:35 } \\
\text { B: 09:55-10:35 } \\
\text { very difficult observing } \\
\text { signal in COR1 }\end{array}$ & $\begin{array}{l}\text { NPCH, L } \\
\alpha(A)=7^{\circ} \\
\beta(A)=6^{\circ} \\
\text { ET }\end{array}$ \\
\hline 21 & $9.46^{\circ}$ & $\begin{array}{l}17121: 48-22: 16 \\
19521: 55 \\
284 \\
30421: 46-21: 56\end{array}$ & $\begin{array}{l}\text { A: } 22: 05-23: 05 \\
\text { B: } 22: 15-23: 05\end{array}$ & $\begin{array}{l}\text { SPCH, L } \\
\alpha(A)=169^{\circ} \\
\beta(A)=165^{\circ} \\
\text { ET-Hel? }\end{array}$ \\
\hline 22 & $9.50^{\circ}$ & $\begin{array}{l}17103: 18-03: 41 \\
19503: 25 \\
28403: 26 \\
30403: 16-03: 46\end{array}$ & $\begin{array}{l}\text { A: } 02: 45-04: 45 \\
\text { B: } 03: 05-03: 55\end{array}$ & $\begin{array}{l}\text { SPCH, L } \\
\alpha(A)=182^{\circ} \\
\beta(A)=185^{\circ} \\
\text { ET-Hel }\end{array}$ \\
\hline 23 & $9.92^{\circ}$ & $\begin{array}{l}171 \text { 19:38-19:58 } \\
195 \text { 19:45-19:55 } \\
284 \text { 19:46 } \\
304 \text { 19:46- 19:56 }\end{array}$ & $\begin{array}{l}\text { A: } 20: 05-21: 15 ? \\
\text { B: } 20: 05-21: 25\end{array}$ & $\begin{array}{l}\text { NPCH, L } \\
\alpha(A)=2^{\circ} \\
\beta(A)=0^{\circ} \\
\text { ET-Hel }\end{array}$ \\
\hline 24 & $10.11^{\circ}$ & $\begin{array}{l}17123: 46-00: 08 \\
19523: 45-00: 15 \\
284 \\
30423: 56-00: 16\end{array}$ & $\begin{array}{l}\text { A: } 00: 15-00: 45 \\
\text { B: }\end{array}$ & $\begin{array}{l}\mathrm{SPCH}, \mathrm{L} \\
\alpha(A)=176^{\circ} \\
\beta(A)=175^{\circ} \\
?\end{array}$ \\
\hline 25 & $11.65^{\circ}$ & $\begin{array}{l}17104: 58-05: 43 \\
19504: 55-05: 55 \\
28405: 06-05: 46 \\
30405: 06-05: 56\end{array}$ & $\begin{array}{l}\text { A: } 05: 25-07: 05 \\
\text { B: } 05: 25-07: 05\end{array}$ & $\begin{array}{l}\text { NPCH, I } \\
\alpha(A)=7^{\circ} \\
\beta(A)=12^{\circ} \\
\text { ET-Hel }\end{array}$ \\
\hline 26 & $12.81^{\circ}$ & $\begin{array}{l}171 \text { not clear } \\
19519: 25-20: 15 \\
28419: 26 \\
30419: 26-19: 46\end{array}$ & $\begin{array}{l}\text { A: } \\
\text { B: } 19: 55-20: 25\end{array}$ & $\begin{array}{l}\text { NPCH, I } \\
\alpha(B)=0^{\circ} \\
\beta(B)=2^{\circ} \\
\text { ET }\end{array}$ \\
\hline
\end{tabular}




\begin{tabular}{|c|c|c|c|c|}
\hline $\mathrm{N}^{\circ}$ & $\begin{array}{l}\text { Date } \\
\Delta \phi_{\mathrm{AB}}\end{array}$ & EUVI & COR1 & $\begin{array}{l}\text { Position } \\
\text { Morphology }\end{array}$ \\
\hline 27 & 2007-07-24 & $\begin{array}{l}17100: 58-01: 06 \\
19501: 05-01: 15\end{array}$ & $\begin{array}{l}\text { A: } 01: 15-02: 05 \\
\text { B: } 01: 15-02: 35\end{array}$ & $\begin{array}{l}\mathrm{NPCH}, \mathrm{I} \\
\alpha(B)=359^{\circ}\end{array}$ \\
\hline & $20.57^{\circ}$ & $\begin{array}{l}284 \\
30400: 56-01: 26\end{array}$ & $\begin{array}{l}\text { very faint } \\
\text { in COR1A }\end{array}$ & $\begin{array}{l}\beta(B)=356^{\circ} \\
\text { ?-Hel? }\end{array}$ \\
\hline 28 & 2007-08-04 & $\begin{array}{l}17120: 38-20: 48 \\
19520: 45 \\
28420: 46 \\
30420: 36-20: 46\end{array}$ & $\begin{array}{l}\text { A: } 20: 55-22: 15 \\
\text { B: }\end{array}$ & $\begin{array}{l}\text { NPCH, L } \\
\alpha(A)=1^{\circ} \\
\beta(A)=0^{\circ} \\
\text { ET }\end{array}$ \\
\hline 29 & $24.63^{\circ}$ & $\begin{array}{l}17101: 08-01: 26 \\
19501: 15-01: 25 \\
28401: 06 \\
30401: 16-01: 36\end{array}$ & $\begin{array}{l}\text { A: } 01: 35-02: 45 \\
\text { B: } 01: 35-03: 06\end{array}$ & $\begin{array}{l}\mathrm{NPCH}, \mathrm{I} \\
\alpha(A)=356^{\circ} \\
\beta(A)=351^{\circ} \\
\text { ET }\end{array}$ \\
\hline 30 & 2007-09-03 & $\begin{array}{l}171 \text { 08:36-09:01 } \\
195 \text { 08:35-08:55 } \\
284 \text { 08:46 } \\
304 \text { 08:36-09:06 }\end{array}$ & $\begin{array}{l}\text { A: } \\
\text { B: } 08: 55-10: 05 ?\end{array}$ & $\begin{array}{l}\text { NPCH, L } \\
\alpha(B)=1^{\circ} \\
\beta(B)=359^{\circ} \\
\text { ET-Hel }\end{array}$ \\
\hline 31 & 2007-09-07 & $\begin{array}{l}171 \text { 16:16-16:46 } \\
19516: 25-16: 35 \\
28416: 26 \\
30416: 16-16: 46\end{array}$ & $\begin{array}{l}\text { A: } 16: 40-17: 15 \\
\text { B: } 16: 40-17: 20 \\
\text { faint in A }\end{array}$ & $\begin{array}{l}\text { NPCH, EDGE } \\
\alpha(B)=18^{\circ} \\
\beta(B)=26^{\circ} \\
\text { Lambda-Hel }\end{array}$ \\
\hline 32 & $30.04^{\circ}$ & $\begin{array}{l}171 \text { 13:08-14:16 } \\
19513: 05-14: 15 \\
28413: 26-13: 46 \\
304 \text { 13:36-13:56 }\end{array}$ & $\begin{array}{l}\text { A: } 13: 35-15: 00 \\
\text { B: } 13: 35-15: 05\end{array}$ & $\begin{array}{l}\text { NPCH, L/I } \\
\alpha(A)=10^{\circ} \\
\beta(A)=16^{\circ} \\
\text { ET-Hel }\end{array}$ \\
\hline 33 & 2007-09-11 & $\begin{array}{l}17112: 18-12: 56 \\
19512: 25-12: 55 \\
28412: 26-12: 46 \\
30412: 16-12: 56\end{array}$ & $\begin{array}{l}\text { A: } 12: 55-14: 05 \\
\text { B: } 12: 45-14: 35\end{array}$ & $\begin{array}{l}\text { NPCH, EDGE } \\
\alpha(A)=26^{\circ} \\
\beta(A)=42^{\circ} \\
?\end{array}$ \\
\hline 34 & $31.17^{\circ}$ & $\begin{array}{l}171 \text { 14:46-14:56 } \\
19514: 55 \\
28414: 46 \\
30414: 46-14: 56\end{array}$ & $\begin{array}{l}\text { A: } 15: 05-15: 45 \\
\text { B: } 15: 05-16: 05\end{array}$ & $\begin{array}{l}\text { SPCH, EDGE/L } \\
\alpha(A)=164^{\circ} \\
\beta(A)=153^{\circ} \\
\text { Lambda }\end{array}$ \\
\hline 35 & 2007-09-23 & $\begin{array}{l}17111: 26-11: 50 \\
19511: 36-11: 55 \\
28411: 26-11: 46 \\
30411: 26-11: 56\end{array}$ & $\begin{array}{l}\text { A: } 12: 05-13: 15 \\
\text { B: } 11: 45-13: 15\end{array}$ & $\begin{array}{l}\mathrm{NPCH}, \mathrm{I} / \mathrm{L} \\
\alpha(A)=346^{\circ} \\
\beta(A)=331^{\circ} \\
\text { Lambda }\end{array}$ \\
\hline 36 & 2007-09-28 & $\begin{array}{l}17111: 06-11: 16 \\
19511: 05-11: 15\end{array}$ & $\begin{array}{l}\text { A: } 11: 25-12: 25 \\
\text { B: } 11: 20-12: 50\end{array}$ & $\begin{array}{l}\mathrm{NPCH}, \mathrm{EDGE} / \mathrm{L} \\
\alpha(A)=344^{\circ}\end{array}$ \\
\hline & $33.46^{\circ}$ & $\begin{array}{l}28411: 06-11: 26 \\
30411: 06-11: 16\end{array}$ & & $\begin{array}{l}\beta(A)=332^{\circ} \\
\text { ET-Hel }\end{array}$ \\
\hline 37 & 2007-10-01 & $\begin{array}{l}17123: 26-23: 36 \\
19523: 25 \\
28423: 26 \\
30423: 26-23: 36\end{array}$ & $\begin{array}{l}\text { A: } 23: 30-00: 30 \\
\text { B: }\end{array}$ & $\begin{array}{l}\mathrm{NPCH}, \mathrm{L} \\
\alpha(A)=359^{\circ} \\
\beta(A)=352^{\circ} \\
?\end{array}$ \\
\hline
\end{tabular}




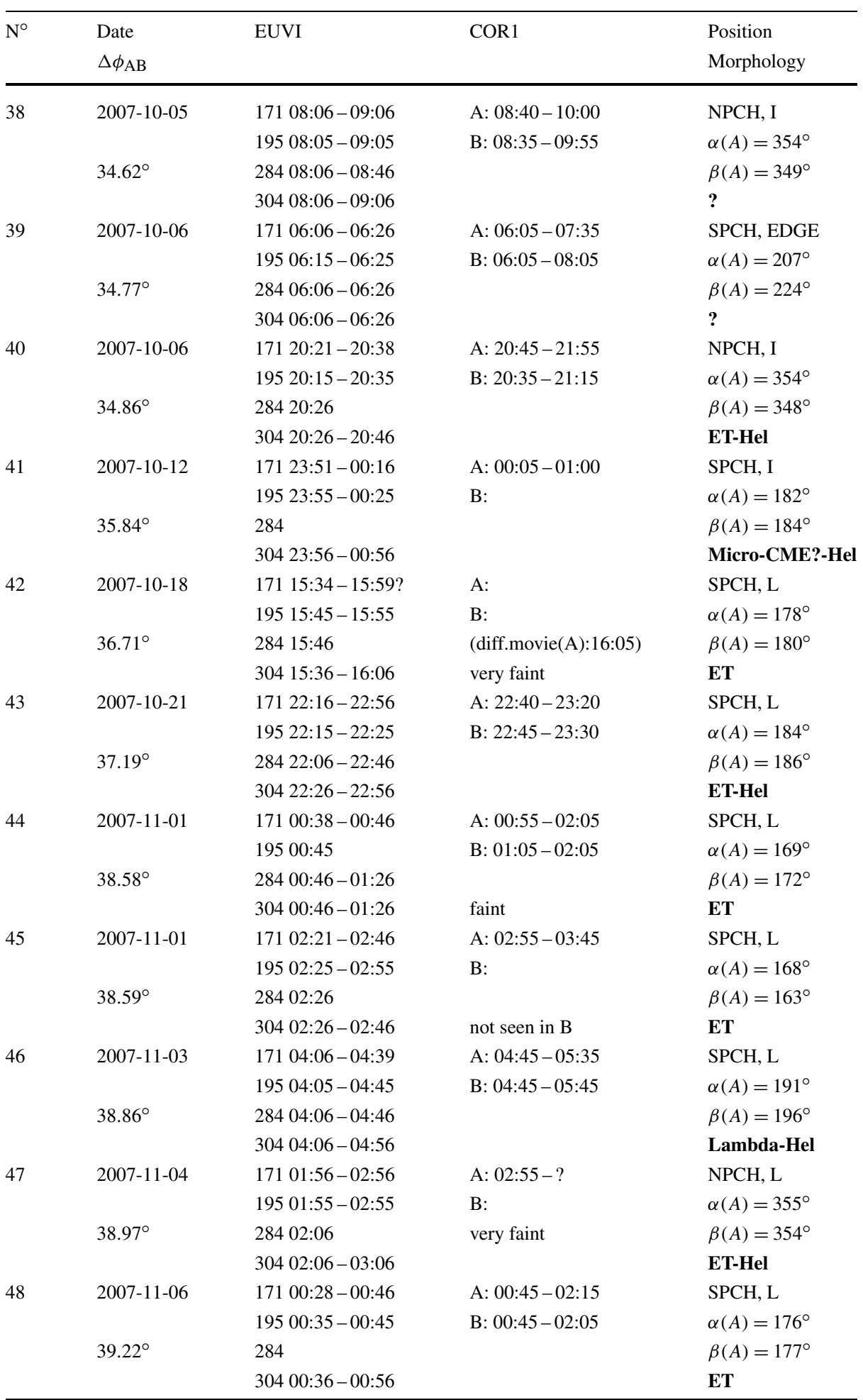




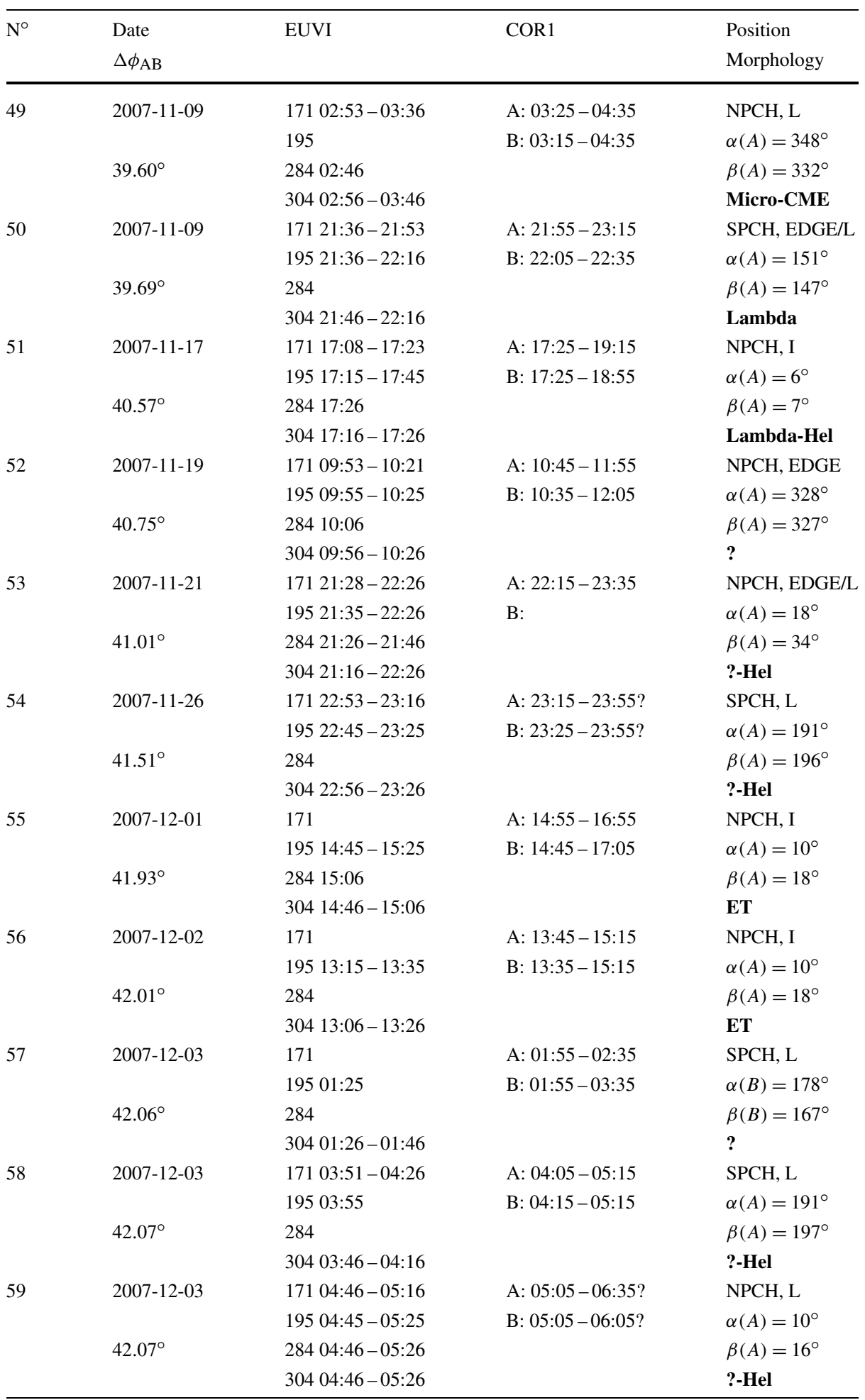




\begin{tabular}{|c|c|c|c|c|}
\hline $\mathrm{N}^{\circ}$ & $\begin{array}{l}\text { Date } \\
\Delta \phi_{\mathrm{AB}}\end{array}$ & EUVI & COR1 & $\begin{array}{l}\text { Position } \\
\text { Morphology }\end{array}$ \\
\hline \multirow[t]{2}{*}{60} & 2007-12-04 & $\begin{array}{l}17101: 06-01: 21 \\
19500: 55-01: 05\end{array}$ & $\begin{array}{l}\text { A:not visible } \\
\text { B: 01:15-02:00 }\end{array}$ & $\begin{array}{l}\text { SPCH, L } \\
\alpha(B)=180^{\circ}\end{array}$ \\
\hline & $42.14^{\circ}$ & $\begin{array}{l}284 \\
30401: 06-01: 36\end{array}$ & $\begin{array}{l}\text { faint } \\
\text { (diff.movie(B):01:25) }\end{array}$ & $\begin{array}{l}\beta(B)=181^{\circ} \\
\text { ET }\end{array}$ \\
\hline \multirow[t]{2}{*}{61} & $2007-12-05$ & $\begin{array}{l}17110: 53-11: 26 \\
19511: 05-11: 25\end{array}$ & $\begin{array}{l}\text { A: } 11: 25-12: 45 \\
\text { B: } 11: 15-12: 55\end{array}$ & $\begin{array}{l}\text { NPCH, I } \\
\alpha(A)=7^{\circ}\end{array}$ \\
\hline & $42.26^{\circ}$ & $\begin{array}{l}284 \\
30410: 56-11: 26\end{array}$ & & $\begin{array}{l}\beta(A)=9^{\circ} \\
\text { ?-Hel }\end{array}$ \\
\hline \multirow[t]{2}{*}{62} & $2007-12-06$ & $\begin{array}{l}171 \text { 15:31-16:09 } \\
19515: 05-15: 35\end{array}$ & $\begin{array}{l}\text { A: } \\
\text { B: }\end{array}$ & $\begin{array}{l}\mathrm{NPCH}, \mathrm{I} \\
\alpha(B)=352^{\circ}\end{array}$ \\
\hline & $42.36^{\circ}$ & $\begin{array}{l}284 \\
30415: 16-16: 16\end{array}$ & (diff.movie(B):16:05) & $\begin{array}{l}\beta(B)=348^{\circ} \\
\text { ET-Hel }\end{array}$ \\
\hline \multirow[t]{2}{*}{63} & $2007-12-06$ & $\begin{array}{l}171 \text { 19:21-19:36 } \\
195 \text { 19:45 }\end{array}$ & $\begin{array}{l}\text { A: } \\
\text { B: }\end{array}$ & $\begin{array}{l}\text { NPCH, L } \\
\alpha(B)=355^{\circ}\end{array}$ \\
\hline & $42.37^{\circ}$ & $\begin{array}{l}284 \text { 19:26 } \\
304 \text { 19:26-19:46 }\end{array}$ & (diff.movie(B):19:45 - 20:05) & $\begin{array}{l}\beta(B)=351^{\circ} \\
?\end{array}$ \\
\hline \multirow[t]{2}{*}{64} & $2007-12-09$ & $\begin{array}{l}17107: 56 \\
19507: 55-08: 25\end{array}$ & $\begin{array}{l}\text { A: } 08: 15-10: 15 \\
\text { B: } 08: 25-09: 45\end{array}$ & $\begin{array}{l}\mathrm{SPCH}, \mathrm{L} \\
\alpha(A)=174^{\circ}\end{array}$ \\
\hline & $42.57^{\circ}$ & $\begin{array}{l}284 \\
30407: 56-08: 26\end{array}$ & & $\begin{array}{l}\beta(A)=172^{\circ} \\
\text { ET }\end{array}$ \\
\hline \multirow[t]{2}{*}{65} & $2007-12-11$ & $\begin{array}{l}171 \text { 19:56-20:13 } \\
19519: 35-19: 45\end{array}$ & $\begin{array}{l}\text { A: } 20: 05-21: 00 ? \\
\text { B: } 20: 05-21: 05\end{array}$ & $\begin{array}{l}\mathrm{NPCH}, \mathrm{L} \\
\alpha(A)=0^{\circ}\end{array}$ \\
\hline & $42.76^{\circ}$ & $\begin{array}{l}284 \text { 20:06 } \\
304 \text { 18:46-20:16 }\end{array}$ & & $\begin{array}{l}\beta(A)=0^{\circ} \\
?\end{array}$ \\
\hline \multirow[t]{2}{*}{66} & $2007-12-12$ & $\begin{array}{l}17121: 56-22: 38 \\
19521: 55\end{array}$ & $\begin{array}{l}\text { A: } \\
\text { B: }\end{array}$ & $\begin{array}{l}\text { SPCH, L } \\
\alpha(A)=178^{\circ}\end{array}$ \\
\hline & $42.84^{\circ}$ & $\begin{array}{l}28422: 06 \\
30421: 56-22: 46\end{array}$ & $\begin{array}{l}\text { not visible } \\
\text { (diff.movie(A):22:25) }\end{array}$ & $\begin{array}{l}\beta(A)=177^{\circ} \\
\text { ET-Hel }\end{array}$ \\
\hline \multirow[t]{2}{*}{67} & $2007-12-12$ & $\begin{array}{l}17122: 21-22: 46 ? \\
19522: 25-22: 46\end{array}$ & $\begin{array}{l}\text { A: } \\
\text { B: }\end{array}$ & $\begin{array}{l}\text { NPCH, EDGE } \\
\alpha(A)=31^{\circ}\end{array}$ \\
\hline & $42.84^{\circ}$ & $\begin{array}{l}28422: 26 \\
30422: 26-22: 56\end{array}$ & $\begin{array}{l}\text { (diff.movie(A):23:05) } \\
\text { faint signature }\end{array}$ & $\begin{array}{l}\beta(A)=56^{\circ} \\
\text { Lambda }\end{array}$ \\
\hline 68 & $2007-12-17$ & $\begin{array}{l}17104: 11-04: 28 \\
19504: 15-04: 45 \\
28404: 26 \\
30404: 16-04: 46\end{array}$ & $\begin{array}{l}\text { A: } \\
\text { B: } \\
(\text { diff.movie(A):04:25- } \\
05: 05)\end{array}$ & $\begin{array}{l}\text { SPCH, EDGE } \\
\alpha(A)=201^{\circ} \\
\beta(A)=216^{\circ} \\
\text { ET }\end{array}$ \\
\hline \multirow[t]{2}{*}{69} & $2007-12-21$ & $\begin{array}{l}17104: 48-05: 03 \\
19504: 55-05: 15\end{array}$ & $\begin{array}{l}\text { A: } \\
\text { B: } 05: 15-06: 25\end{array}$ & $\begin{array}{l}\text { NPCH, I } \\
\alpha(B)=353^{\circ}\end{array}$ \\
\hline & $43.40^{\circ}$ & $\begin{array}{l}284 \\
30404: 46-05: 16\end{array}$ & & $\begin{array}{l}\beta(B)=351^{\circ} \\
\text { ET-Hel }\end{array}$ \\
\hline \multirow[t]{2}{*}{70} & $2007-12-22$ & $\begin{array}{l}17103: 03-03: 38 \\
19503: 05-03: 35\end{array}$ & $\begin{array}{l}\text { A: } \\
\text { B: } 03: 20-04: 50\end{array}$ & $\begin{array}{l}\mathrm{NPCH}, \mathrm{L} \\
\alpha(B)=351^{\circ}\end{array}$ \\
\hline & $43.46^{\circ}$ & $\begin{array}{l}28403: 26 \\
30403: 06-03: 46\end{array}$ & & $\begin{array}{l}\beta(B)=347^{\circ} \\
\text { ET-Hel }\end{array}$ \\
\hline
\end{tabular}




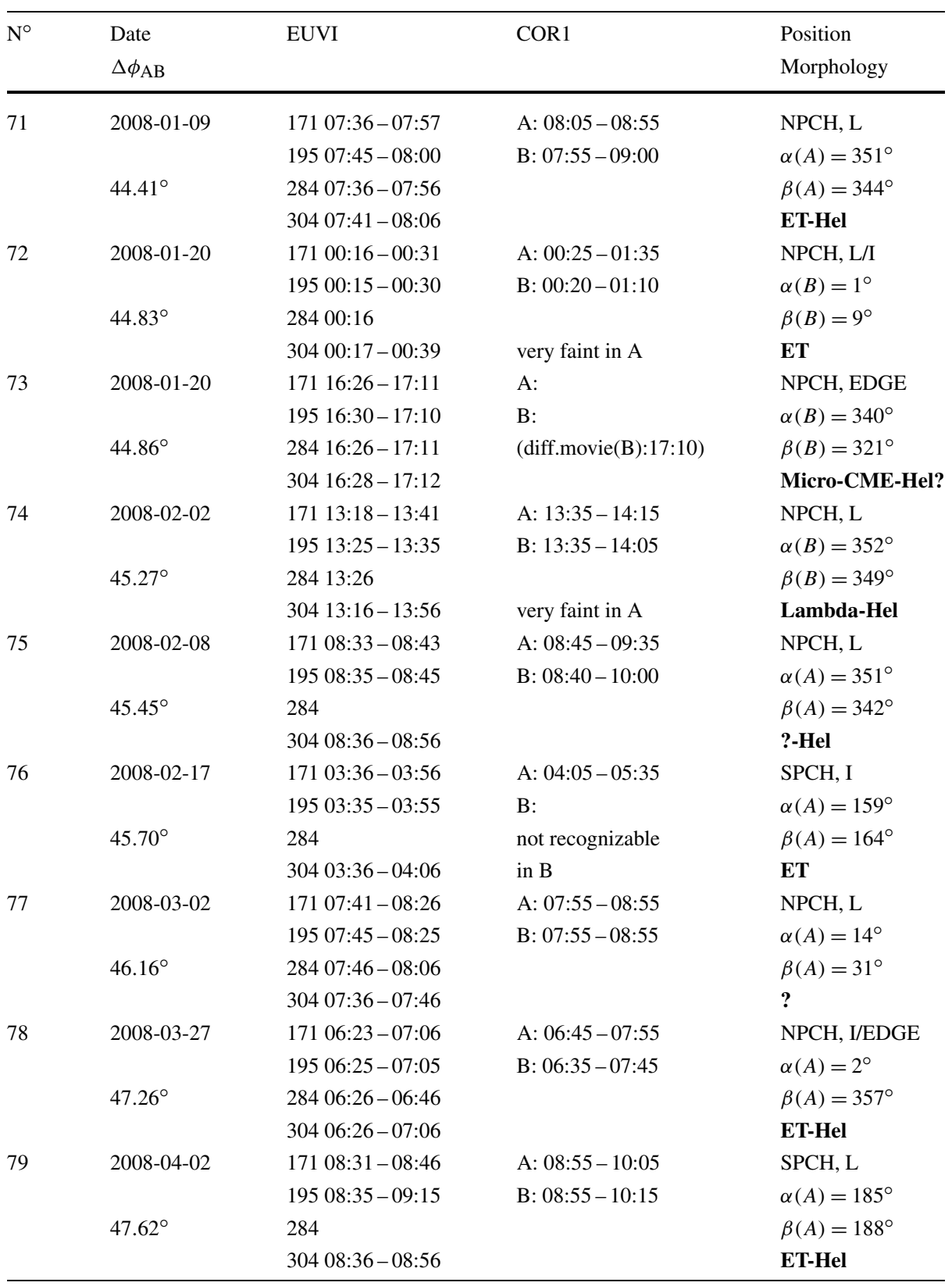

\section{References}

Alexander, D., Fletcher, L.: 1999, Solar Phys. 190, 167.

Cirtain, J., Golub, L., Lundquist, L., van Ballegooijen, A., Savcheva, A., Shimojo, M., DeLuca, E., Tsuneta, S., Sakao, T., Revves, K., Weber, M., Kano, R., Nakurage, N., Shibasaki, K.: 2007, Science 318, 1580.

Cremades, H., Bothmer, V.: 2004, Astron. Astrophys. 422, 307. 
Culhane, L., Harra, L.K., Baker, D., van Driel-Gesztelyi, L., Sun, J., Dosheck, G.A., Brooks, D.H., Lundquist, L.L., Kamio, S., Young, P.R., Hansteen, V.H.: 2007, Publ. Astron. Soc. Japan 59, S751.

Filippov, B., Golub, L., Koutchmy, S.: 2009, Solar Phys. 254, 259.

Gilbert, H.R., Holzer, T.E., Burkepile, J.T., Hundhausen, A.J.: 2000, Astrophys. J. 537, 503.

Howard, T.A., Tappin, S.J.: 2008, Solar Phys. 252, 373.

Howard, R.A., Moses, J.D., Vourlidas, A., Newmark, J.S., Socker, D.G., Plunkett, S.P., Korendyke, C.M., Cook, J.W., Hurley, A., Davila, J.M., Thompson, W.T., St. Cyr, O.C., Mentzell, E., Mehalick, K., Lemen, J.R., Wüelser, J.P., Duncan, D.W., Tarbell, T.D., Wolfson, C.J., Moore, A., Harrison, R.A., Waltham, N.R., Lang, J., Davis, C.J., Eyles, C.J., Mapson-Menard, H., Simnett, G.M., Halain, J.P., Defise, J.M., Mazy, E., Rochus, P., Mercier, R., Ravet, M.F., Delmotte, F., Auchère, F., Delaboudinière, J.P., Bothmer, V., Deutsch, W., Wang, D., Rich, N., Cooper, S., Stephens, V., Maahs, G., Baugh, R., McMullin, D., Carter, T.: 2008, Space Sci. Rev. 136, 67.

Kaiser, M.L., Kucera, T.A., Davila, J.M., St. Cyr, O.C., Guhathakurta, M., Christian, E.: 2008, Space Sci. Rev. 136, 5.

Kamio, S., Hara, H., Watanabe, T., Matsuzaki, K., Shibata, K., Culhane, L., Warren, H.P.: 2007, Publ. Astron. Soc. Japan 59, S757.

Moreno-Insertis, F., Galsgaard, K., Ugarte-Urra, I.: 2008, Astrophys. J. 673, L211.

Pariat, E., Antiochios, S.K., DeVore, C.R.: 2009, Astrophys. J. 691, 16.

Patsourakos, S., Pariat, E., Vourlidas, A., Antiochios, S.K., Wuelser, J.P.: 2008, Astrophys. J. 680, L73.

Raouafi, N.-E., Petrie, G.J.D., Norton, A.A., Henney, C.J., Solanki, S.K.: 2008, Astrophys. J. 682, L137.

Savcheva, A., Cirtain, J., Deluca, E.E., Lundquist, L.L., Golub, L., Weber, M., Shimojo, M., Shibasaki, K., Sakao, T., Narukage, N., Tsuneta, S., Kano, R.: 2007, Publ. Astron. Soc. Japan 59, S771.

Shibata, K., Ishido, Y., Acton, L.W., Strong, K.T., Hirayama, T., Uchida, Y., McAllister, A.H., Matsumoto, R., Tsuneta, S., Shimizu, T., Hara, H., Sakurai, T., Ichimoto, K., Nishino, Y., Ogawara, Y.: 1992, Publ. Astron. Soc. Japan 44, L173.

Shimojo, M., Shibata, K.: 2000, Astrophys. J. 542, 1100.

Shimojo, M., Hashimoto, S., Shibata, K., Hirayama, T., Hudson, H.S., Acton, L.W.: 1996, Publ. Astron. Soc. Japan 48, 123.

Wang, Y.-M., Sheeley, Jr. N.R., Socker, D.G., Howard, R.A., Brueckner, G.E., Michels, D.J., Moses, D., St. Cyr, O.C., Llbaria, A., Delaboudiniére, J.-P.: 1998, Astrophys. J. 508, 899.

Yamauchi, Y., Moore, R.L., Suess, S.T., Wang, H., Sakurai, T.: 2004, Astrophys. J. 605, 511.

Yokoyama, T., Shibata, K.: 1995, Nature 375, 42.

Yokoyama, T., Shibata, K.: 1996, Publ. Astron. Soc. Japan 48, 353. 